\title{
Elevated STC-1 augments the invasiveness of triple-negative breast cancer cells through activation of the JNK/c-Jun signaling pathway
}

\author{
JEONGHUN HAN ${ }^{1,3^{*}}$, MYEONGJIN JEON ${ }^{1,2 *}$, INCHEOL SHIN ${ }^{3}$ and SANGMIN KIM ${ }^{1}$ \\ ${ }^{1}$ Department of Surgery, Samsung Medical Center, Seoul; ${ }^{2}$ Department of Health Sciences and Technology, \\ SAIHST, Sungkyunkwan University, Seoul; ${ }^{3}$ Department of Life Science, \\ Hanyang University, Seoul, Republic of Korea
}

Received January 5, 2016; Accepted March 2, 2016

DOI: $10.3892 /$ or.2016.4977

\begin{abstract}
Stanniocalcin-1 (STC-1), a secreted glycoprotein, is highly expressed in a variety of human malignancies. However, the role of STC-1 has not been fully elucidated in breast cancer cells. Here, we investigated whether STC-1 acts as a prognostic factor in triple-negative breast cancer (TNBC) patients, and we explored the cellular mechanism in breast cancer cells. The level of STC-1 expression was directly associated with the relapse-free and overall survival of basal-type breast cancer patients. Breast cancer patients with a high level of STC-1 had poor prognosis. In addition, our results showed that the level of STC-1 expression was markedly higher in TNBC than in non-TNBC cells. Invasiveness of the TNBC cells was also significantly increased in response to recombinant human STC-1 treatment. In contrast, the invaded cell numbers were completely decreased by STC-1 siRNA overexpression in the Hs578T and MDA-MB-231 TNBC cells. Our results showed that the phosphorylation of c-Jun N-terminal protein kinase (JNK) and c-Jun was increased after STC-1 treatment but not the phosphorylation of ERK and p38 MAPKs in the Hs578T and MDA-MB-231 TNBC cells. Furthermore, expression of one invasion-related gene MMP-9, was increased by STC-1 treatment. STC-1-induced MMP-9 expression was suppressed by SP600125 (a JNK inhibitor) in the Hs578T cells. STC-1-induced cell invasiveness was also
\end{abstract}

Correspondence to: Dr Sangmin Kim, Department of Surgery, Samsung Medical Center, 50 Irwon-dong, Gangnam-gu, Seoul 06351, Republic of Korea

E-mail: sangmin3005.kim@samsung.com

Professor Incheol Shin, Department of Life Science, Hanyang University, 222 Wangshimni-ro, Seoul 04769, Republic of Korea

E-mail: incheol@hanyang.ac.kr

*Contributed equally

Key words: stanniocalcin-1, prognosis, triple-negative breast cancer, cell invasion, JNK inhibited by SP600125. Taken together, we demonstrated that aberrant STC-1 expression is associated with poor prognosis and stimulates the invasiveness of TNBC cells through the JNK/c-Jun-dependent signaling pathway.

\section{Introduction}

Breast cancer is the most commonly diagnosed malignancy and the leading cause of death from cancer among women and the incidence rate is higher in young women in comparison to older ones $(1,2)$. One breast cancer subtype, triple-negative breast cancer (TNBC) accounts for $\sim 15-20 \%$ of breast cancer cases and occurs more commonly in younger women $(3,4)$. TNBCs are highly aggressive and often have a poor prognosis when compared to other breast cancer subtypes, including luminal and HER2-type breast cancers $(5,6)$. Due to the lack of ER, PR, and HER 2 expression, TNBCs are insensitive to anti-hormonal and HER2-targeted therapies (7). Although sensitive to chemotherapy, TNBCs have an intrinsic aggressive clinical course associated with high proliferation, high histologic grade, earlier time to recurrence, and higher risk of distant recurrence (5). Thus, many researchers have attempted to discover promising therapeutic targets for the treatment of TNBC breast cancer patients.

Stanniocalcins (STCs) are secreted glycoprotein hormones and are involved in calcium/phosphate homeostasis and cell development $(8,9)$. STCs consist of STC-1 and -2 and are broadly expressed in multiple mammalian tissues including the ovary, prostate, and kidney $(8,10,11)$. In particular, STC-1, a 56-kDa homodimeric glycoprotein hormone, is highly expressed in a variety of cancers such as breast, ovarian, and colorectal cancers (12-15). STC-1 is involved in various biological events such as cell proliferation, apoptosis, and inflammation $(14,16)$. Furthermore, STC-1 triggers tumor angiogenesis via upregulation of vascular endothelial growth factor (VEGF) in gastric cancer cells (17). Abnormal STC-1 expression is generally correlated with tumorigenesis and poor clinical outcomes in ovarian and colorectal cancers $(10,14,18)$. However, little is understood about the clinical significance of STC-1 expression and the intracellular signaling events in breast cancer. 
Ninety percent of all cancer-related deaths are caused by tumor metastasis. Metastasis is a process which includes excessive tumor cell proliferation, degradation of the surrounding stroma microenvironment [extracellular matrix (ECM)], migration, and invasion $(19,20)$. In this multiple-step process, the biological activity of matrix metalloproteinases (MMPs), a large family of ECM-degrading enzymes, facilitates tumor cell growth, migration, and angiogenesis through cleavage of ECM components (21). The expression of MMPs in tumors is primarily regulated at the transcriptional level in response to growth factors and cytokines $(22,23)$. Enhancement of MMP-1 expression by TNF- $\alpha$ is mediated by coordinate activation of c-Jun N-terminal protein kinase (JNK) and p38 MAPK pathways (24). In particular, the JNK/c-Jun signaling pathway accelerates cell migration and invasion and promotes metastasis in breast cancer cells (25-28). In contrast, Fyn-related kinase (FRK) was found to reduce cell migration and invasion via inhibiting the JNK/c-Jun signaling pathway in glioma cells (28).

The aim of this study was to investigate the clinical significance of STC-1 in basal-type breast cancer as well as the regulatory mechanism of STC-1-induced cell invasion. Consequentially, elevated STC-1 expression was associated with poor prognosis in basal-type breast cancer patients. The levels of STC-1 mRNA and protein expression were also significantly increased in the TNBC cells. Furthermore, recombinant human STC-1 treatment augmented the invasiveness of the TNBC cells while STC-1 siRNA overexpression suppressed the invasiveness of the TNBC cells. In addition, we found for the first time that STC-1 upregulated MMP-9 expression through the JNK/c-Jun-dependent signaling pathway. STC-1-induced MMP-9 expression was associated with the invasiveness of the TNBC cells. Therefore, we demonstrated that aberrant STC-1 expression enhanced the invasiveness of the TNBC cells through JNK/c-Jun-dependent MMP-9 induction. STC-1 may be a promising therapeutic target for the treatment of TNBC patients.

\section{Materials and methods}

Reagents. Cell culture media and antibiotics were purchased from Life Technologies (Rockville, MD, USA). Fetal bovine serum (FBS) was purchased from Hyclone (Logan, UT, USA). Anti-phospho-JNK, c-Jun, ERK, p38, and STC-1 antibodies were purchased from Epitomics (Burlingame, CA, USA). Anti-total-JNK, ERK, p38, and $\beta$-actin antibodies were purchased from AbFrontier (Seoul, Korea). Secondary horseradish peroxidase (HRP)-conjugated antibodies were purchased from Santa Cruz Biotechnology (Santa Cruz, CA, USA). Recombinant human STC-1 was purchased from ProSpec (ProSpec HOR-259; Israel). SP600125 was purchased from Calbiochem (San Diego, CA, USA).

Cell cultures and drug treatment. MCF7, Hs578T, MDA-MB-231, and MDA-MB-468 breast cancer cells were cultured in Dulbecco's modified Eagle's medium (DMEM) supplemented with $10 \% \mathrm{FBS}, 100 \mathrm{IU} / \mathrm{ml}$ penicillin, and $100 \mu \mathrm{g} / \mathrm{ml}$ streptomycin. BT474 and SKBR3 breast cancer cells were cultured in RPMI-1640 supplemented with $10 \%$ FBS, $100 \mathrm{IU} / \mathrm{ml}$ penicillin, and $100 \mu \mathrm{g} / \mathrm{ml}$ streptomycin. Cells were grown in a humidified atmosphere with $5 \% \mathrm{CO}_{2}$ at $37^{\circ} \mathrm{C}$. In the drug treatment experiment, after serum starvation for $24 \mathrm{~h}$, the cells were pre-treated with SP600125 for $1 \mathrm{~h}$, and then treated with recombinant human STC-1 for $24 \mathrm{~h}$.

Analysis of public database. Expression data were downloaded from a public database [Kaplan-Meier plotter database (http://kmplot.com/breast)]. The clinical value of STC-1 was analyzed by Kaplan-Meier survival plots in basal-type breast cancer patients. The hazard ratio with $95 \%$ confidence intervals and log-rank P-values were calculated.

Real-time polymerase chain reaction $(P C R)$. Total RNA was extracted from the cells using TRIzol reagent (Invitrogen, Carlsbad, CA, USA), according to the manufacturer's protocol. Isolated RNA samples were then used for RT-PCR. Samples of total RNA $(1 \mu \mathrm{g})$ were reverse-transcribed into cDNA in $20-\mu 1$ reaction volumes using a first-strand cDNA synthesis kit for RT-PCR, according to the manufacturer's instructions (MBI Fermentas, Hanover, MD, USA). Gene expression levels were quantified by real-time PCR using a SensiMix SYBR kit (Bioline, Ltd., London, UK) and $100 \mathrm{ng}$ of cDNA per reaction. The primer sequences used for this analysis were as follows: human STC-1 (forward, 5'-CAC ACC CAC GAG CTG ACT TC-3' and reverse, 5'-TCT CCC TGG TTA TGC ACT CTC-3') and ACTB as an internal control (forward, 5'-TCA CCA TTG GCA ATG AGC GGT T-3' and reverse, 5'-AGT TTC GTG GAT GCC ACA GGA CT-3'). An annealing temperature of $60^{\circ} \mathrm{C}$ was used for all of the primers. PCRs were performed in a standard 384-well plate format with an ABI 7900HT real-time PCR detection system (Applied Biosystems, Foster City, CA, USA). For data analysis, the raw threshold cycle $(\mathrm{Ct})$ value was first normalized to the housekeeping gene for each sample to obtain a $\Delta \mathrm{Ct}$. The normalized $\Delta \mathrm{Ct}$ was then calibrated to the control cell samples resulting in a $\Delta \Delta \mathrm{Ct}$.

Western blotting. Cell lysates were prepared for detection of STC-1, $\beta$-actin, anti-total and phospho-JNK, c-Jun, ERK, p38 expression. Equal proteins $(50 \mu \mathrm{g})$ were boiled for $5 \mathrm{~min}$ in Laemmli sample buffer and then electrophoresed on $10 \%$ sodium dodecyl sulfate-polyacrylamide (SDS-PAGE) gels. Separated proteins were transferred to polyvinylidene fluoride (PVDF) membranes, and the membranes were blocked with $10 \%$ skim milk in Tris-buffered saline (TBS) containing $0.01 \%$ Tween-20 (TBST) for $15 \mathrm{~min}$. The blots were washed three times in TBST and then incubated with STC- $1, \beta$-actin, anti-total and phospho-JNK, c-Jun, ERK, p38 antibodies in TBST buffer at $4^{\circ} \mathrm{C}$ overnight. The blots were washed three times in TBST and subsequently incubated with secondary HRP-conjugated antibodies (1:2,000 dilution) in TBST buffer. After a 1-h incubation at room temperature (RT), the blots were washed three times in TBST. ECL ${ }^{\mathrm{TM}}$ Prime reagent (GE Healthcare, Buckinghamshire, UK) was used for development.

Cell invasion assay. Twenty-four-well Boyden chambers with Matrigel-coated filters ( $8-\mu \mathrm{m}$ pore size) were purchased from Becton-Dickinson (San Diego, CA, USA). Hs578T and MDA-MB-231 breast cancer cells transfected with scrambled 

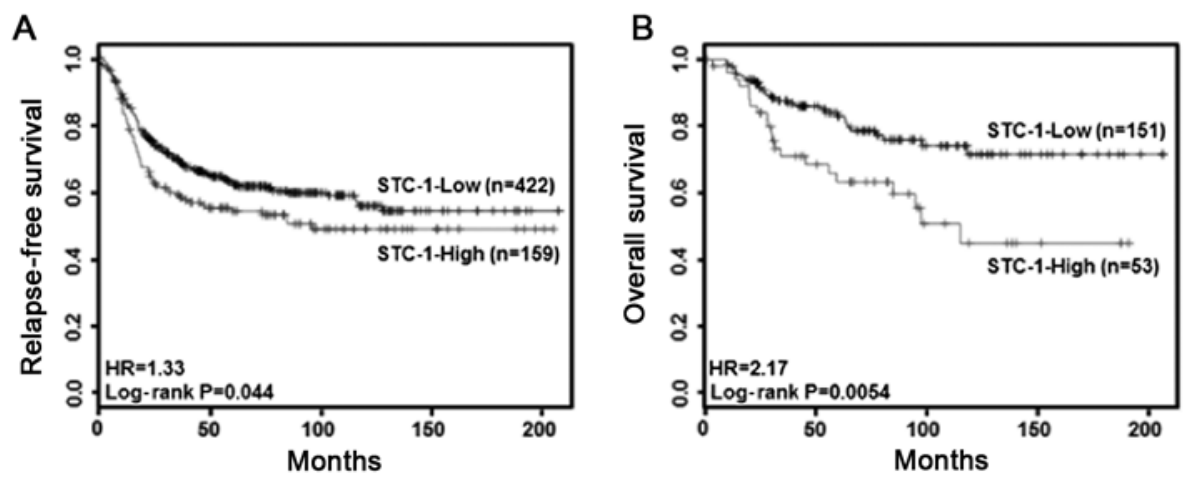

Figure 1. Elevated STC-1 expression is associated with poor prognosis in basal-type breast cancer patients. The expression data for STC-1 were obtained from a public database [Kaplan-Meier plotter database (http://kmplot.com/breast)]. The clinical value of STC-1 was analyzed by Kaplan-Meier survival plot in basal-type breast cancer patients. (A) Relapse-free survival. (B) Overall survival.

and STC-1 siRNA were tested for invasion at $48 \mathrm{~h}$. The cells were resuspended in culture media $\left(5 \times 10^{4}\right.$ cells/well) and were seeded into the Matrigel-coated upper compartment of the invasion chambers. Fresh culture media with 5\% FBS were added to the lower compartment of the invasion chamber. In addition, Hs578T cells were resuspended in culture media and then seeded into the Matrigel-coated upper compartment of the invasion chambers in the presence or absence of $50 \mathrm{ng} / \mathrm{ml}$ STC-1 and/or $10 \mu \mathrm{M}$ SP600125. Fresh culture media with 5\% FBS were added to the lower compartment of the invasion chamber. After $24 \mathrm{~h}$ (Hs578T cells) or $48 \mathrm{~h}$ (MDA-MB-231 cells) of incubation, the cells on the upper side of the filter were removed using cotton swabs. Invaded cells through the Matrigel were located on the underside of the filter. The underside of the filter was fixed in $100 \%$ methanol, washed in $1 \mathrm{X}$ PBS, and stained using hematoxylin and eosin (H\&E). These cells were analyzed using Aperio ScanScope XT (Aperio Technologies, Vista, CA, USA).

STC-1 siRNA transfection. Scrambled and STC-1 siRNAs were purchased from Bioneer (Daejeon, Korea). Hs578T and MDA-MB-231 TNBC cells were seeded in a 6-well plate. Cell transfection was performed using Lipofectamine 2000 (Invitrogen) according to the manufacturer's protocol. The cells were maintained in culture media without FBS and antibiotics for $24 \mathrm{~h}$ while Lipofectamine transfection, and then further incubated in fresh culture media with $10 \%$ FBS for $24 \mathrm{~h}$.

Enzyme-linked immunosorbent assay. ELISA assay was performed on culture media (200 $\mu \mathrm{l})$ collected from Hs578T and MDA-MB-231 TNBC cells. Secreted protein levels of STC-1 were measured using an ELISA kit (R\&D Systems, Minneapolis, MN, USA), according to the manufacturer's instructions. Secreted protein levels were analyzed at a wavelength of $450 \mathrm{~nm}$ on a spectrometer (SpectraMax 190; Molecular Devices, Sunnyvale, CA, USA).

Zymography. Samples (100 $\mu$ l) were resuspended in loading buffer and run on a $10 \%$ SDS-PAGE gel that contained $0.5 \mathrm{mg} / \mathrm{ml}$ gelatin without prior denaturation. After electrophoresis, the gels were washed to remove SDS and incubated for $30 \mathrm{~min}$ at RT in a renaturing buffer. The gels were incubated for $48 \mathrm{~h}$ at $37^{\circ} \mathrm{C}$ in a developing buffer. Next, the gels were subsequently stained with Coomassie Brilliant Blue G-250, destained in 30\% methanol, and flooded with $10 \%$ acetic acid to detect gelatinase secretion.

Statistical analysis. Statistical significance was determined using a Student's t-test. The results are presented as means \pm SEM. All quoted P-values were two-tailed and differences were considered statistically significant when $\mathrm{P}<0.05$. Statistical analysis was performed using Microsoft Excel.

\section{Results}

Elevated STC-1 expression is associated with poor prognosis in basal-type breast cancer patients. Clinically, to investigate the correlation between STC-1 expression and the prognosis of breast cancer patients, we analyzed the clinical value of STC-1 in breast cancer patients using a Kaplan-Meier plotter database (http://kmplot.com/breast). As shown in Fig. 1A and B, we found that breast cancer patients with a high level of STC-1 presented with poor prognosis. In the basal-type breast cancer patients, patients with high expression of STC-1 showed poorer relapse-free $(\mathrm{P}=0.044$, Fig. $1 \mathrm{~A})$ and overall survival $(\mathrm{P}=0.0054$, Fig. 1B) than did patients with low expression. However, the level of STC-1 expression did not affect the relapse-free and overall survival of the luminal- and HER2-type breast cancer patients (data not shown). Therefore, we demonstrated that aberrant STC-1 expression is associated with poor clinical outcomes in basal-type breast cancer patients.

The levels of STC-1 expression in breast cancer cells. Next, we measured the levels of STC-1 mRNA and protein expression in various breast cancer cell lines. Our results showed that the levels of STC-1 expression were higher in the TNBC cells than these levels in the non-TNBC cells (Fig. 2A and B). The levels of STC-1 mRNA expression were significantly increased in the TNBC cells when compared with levels in the non-TNBC cells (Fig. 2A). The levels of STC-1 mRNA expression were significantly increased by $103.16 \pm 9.51$-fold (Hs578T cells), 58.45 \pm 1.71 -fold (MDA-MB-231 cells), and $27.88 \pm 3.94$-fold (MDA-MB-468 cells) of the control level (MCF7 cells) (Fig. 2A). The STC-1 mRNA level of the Hs578T 
A

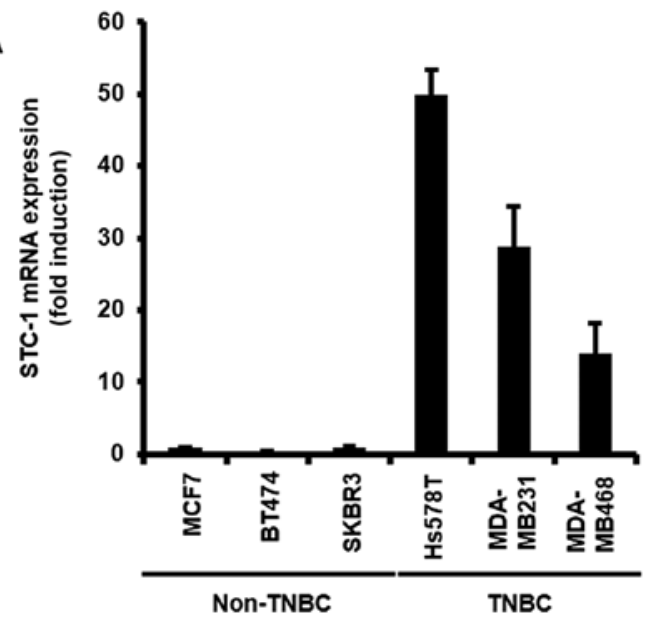

B

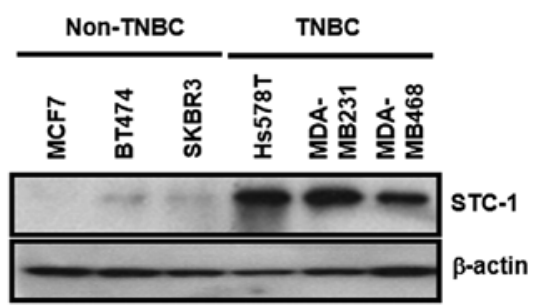

Figure 2. Levels of STC-1 expression in breast cancer cells. (A) The levels of STC-1 mRNA expression were analyzed by real-time PCR. (B) The levels of STC-1 and $\beta$-actin expression were analyzed by western blotting in whole cell lysates. These results are representative of three independent experiments. The values shown are the means \pm SEM.
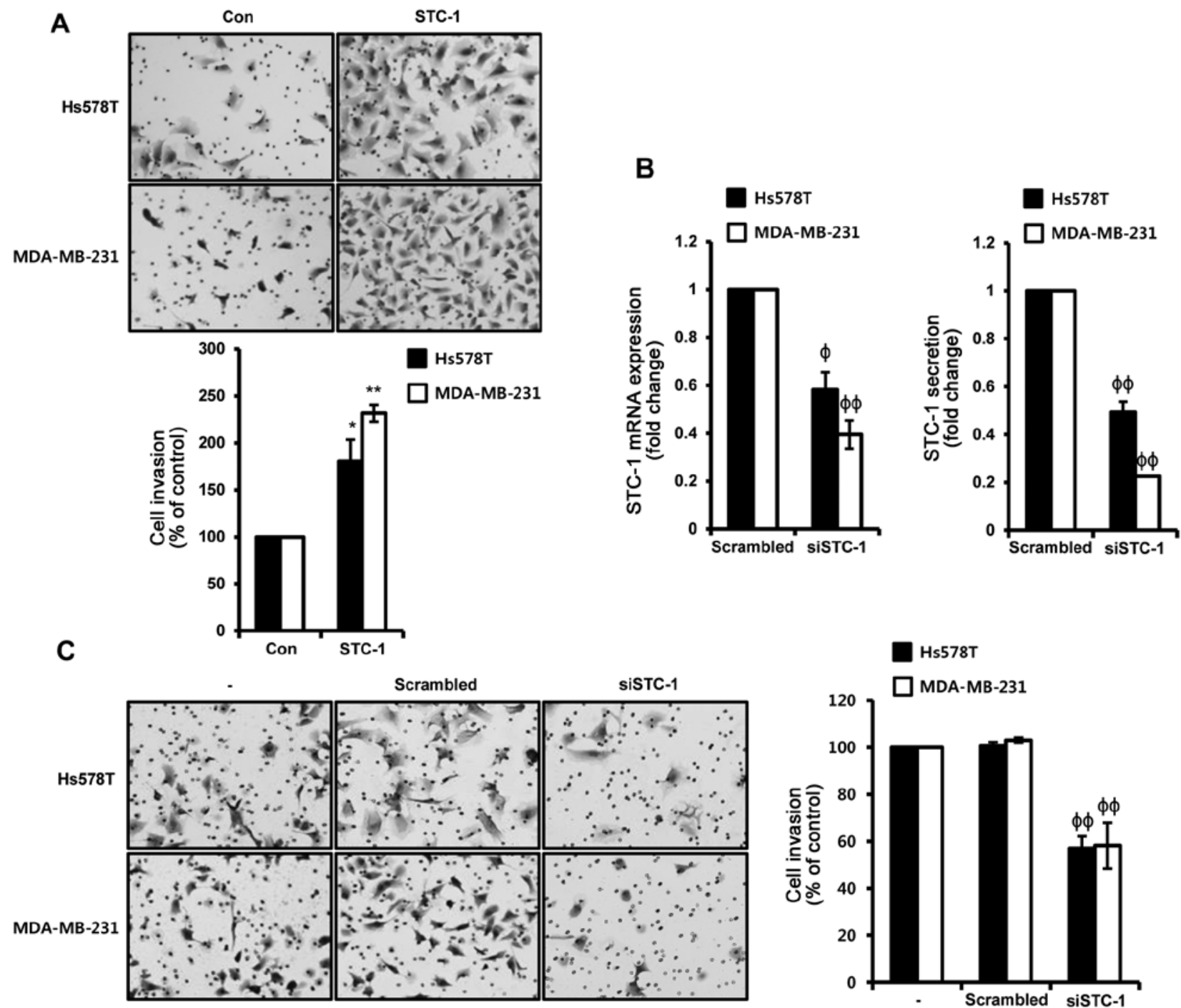

Figure 3. Induction of STC-1 expression enhances the invasiveness of TNBC cells. (A) After serum starvation for $24 \mathrm{~h}$, Hs578T and MDA-MB-231 TNBC cells were treated with $50 \mathrm{ng} / \mathrm{ml} \mathrm{STC}-1$ for 24 and $48 \mathrm{~h}$, respectively. Invasiveness of TNBC cells was analyzed by Boyden chamber assay, as described in 'Materials and methods'. (B and C) Hs578T and MDA-MB-231 TNBC cells were transfected with scrambled and STC-1 siRNA for 48 h, respectively. (B) The levels of STC-1 mRNA and protein secretion were analyzed by real-time PCR (left) and ELISA (right), respectively, in scrambled and STC-1 siRNA overexpressed cells. (C) After transfection for $48 \mathrm{~h}$, invasiveness of scrambled and STC-1 siRNA overexpressed cells was analyzed by Boyden chamber assay, as described in 'Materials and methods'. These results are representative of three independent experiments. The values shown are the means \pm SEM. "P $<0.05$, ${ }^{* *} \mathrm{P}<0.01$ vs. control, ${ }^{\phi} \mathrm{P}<0.05,{ }^{\phi}{ }^{\mathrm{P}}<0.01$ vs. scrambled siRNA overexpressed cells. 
A

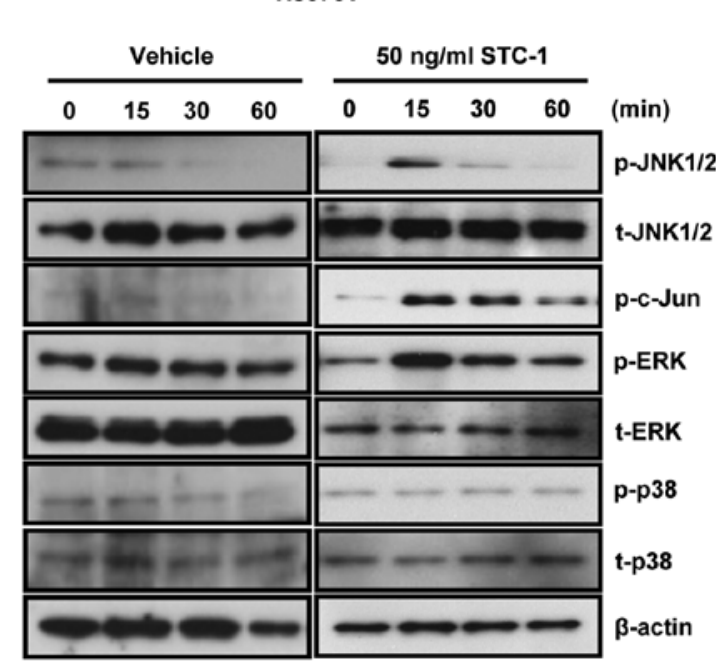

B

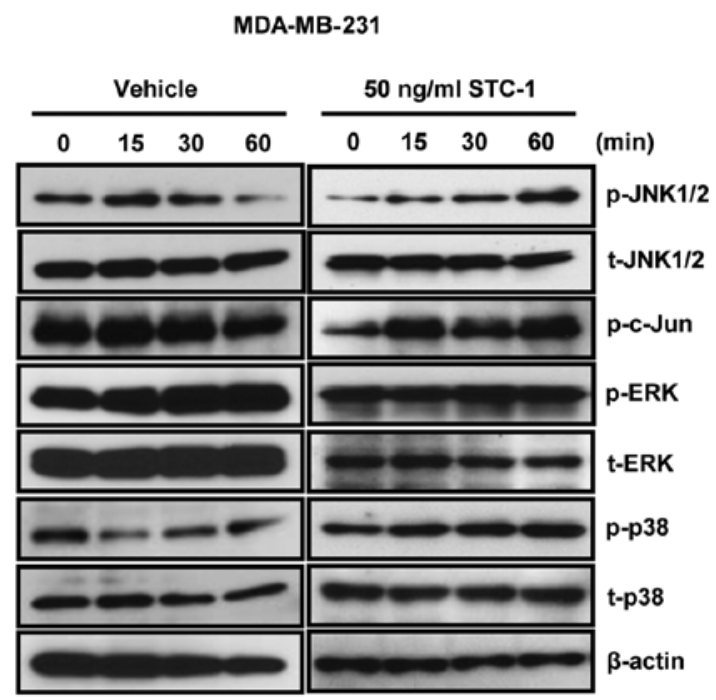

Figure 4. STC-1 activates the phosphorylation of JNK/c-Jun in the TNBC cells. After serum starvation for 24 h, (A) Hs578T and (B) MDA-MB-231 TNBC cells were treated with $50 \mathrm{ng} / \mathrm{ml}$ STC-1 for the indicated times. The levels of $\mathrm{p}$-JNK, $\mathrm{t}-\mathrm{JNK}$, $\mathrm{p}$-c-Jun, $\mathrm{p}$-ERK, $\mathrm{t}$-ERK, $\mathrm{p}$-p 38 , $\mathrm{t}$-p 38 , and $\beta$-actin expression were analyzed by western blotting in whole cell lysates. These results are representative of three independent experiments.

cells was the highest among the TNBC cells (Fig. 2A). Under the same condition, STC-1 protein levels were also increased in the TNBC cells (Fig. 2B). Based on these results, we demonstrated that the levels of STC-1 expression were the highest in the TNBC cells among all the breast cancer cell types.

Induction of STC-1 expression enhances the invasiveness of TNBC cells. To verify the effect of STC-1 on TNBC cells, Hs578T and MDA-MB-231 TNBC cells were treated with $50 \mathrm{ng} / \mathrm{ml} \mathrm{STC}-1$ for 24 and $48 \mathrm{~h}$, respectively. As shown in Fig. 3A, recombinant human STC-1 significantly increased the invasiveness of the TNBC cells. The rates of cell invasion in response to STC-1 treatment were increased by 180.38 and $231.53 \%$, compared to the vehicle-treated Hs578T and MDA-MB-231 cells, respectively (Fig. 3A). In contrast, we investigated whether STC-1 siRNA overexpression suppresses the invasion ability of TNBC cells. Hs578T and MDA-MB-231 cells were transfected with scrambled or STC-1 siRNA for $48 \mathrm{~h}$, respectively. We observed that the levels of STC-1 mRNA and secreted protein were decreased by STC-1 siRNA overexpression in the Hs578T and MDA-MB-231 TNBC cells. The levels of STC-1 mRNA expression were decreased to $0.58 \pm 0.07$-fold and $0.39 \pm 0.06$-fold of the control level in the STC-1 siRNA overexpressed cells, respectively (Fig. 3B, left). Under the same conditions, the levels of STC-1 secreted protein were decreased to $0.49 \pm 0.04$-fold and $0.23 \pm 0.003$-fold of the control level in the STC-1 siRNA overexpressed cells, respectively (Fig. 3B, right). In addition, the invasiveness of TNBC cells was completely suppressed by STC-1 siRNA overexpression (Fig. 3C). The rates of cell invasion by STC-1 silencing were decreased to 56.96 and $58.16 \%$ of the control Hs578T and MDA-MB-231 cells, respectively (Fig. 3C). Based on these results, the levels of STC-1 expression were directly associated with the invasive ability of the TNBC cells.

STC-1 activates the phosphorylation of JNK/c-Jun in TNBC cells. Next, we investigated how JNK/c-Jun regulates the invasiveness of TNBC cells in response to STC-1. After recombinant human STC-1 treatment for the indicated time, whole cell lysates were harvested for detecting the activities of signaling molecules in the Hs578T and MDA-MB-231 TNBC cells. Our results showed that exogenous STC-1 increased the phosphorylation of JNK and c-Jun in both the Hs578T (Fig. 4A) and MDA-MB-231 (Fig. 4B) TNBC cells. The phosphorylation of JNK and c-Jun reached a maximum level $15 \mathrm{~min}$ after STC-1 treatment in the Hs578T cells (Fig. 4A). We also observed that the STC-1-induced phosphorylation of JNK and c-Jun was still activated after $60 \mathrm{~min}$ in the MDA-MB-231 cells (Fig. 4B). The phosphorylation of ERK was activated by STC-1 in the Hs578T cells, whereas the phosphorylation of ERK was not activated by STC-1 in the MDA-MB-231 cells (Fig. 4A and B). The phosphorylation of p38 did not change in response to STC-1 treatment in both the Hs578T and MDA-MB-231 cells (Fig. 4A and B). Therefore, we demonstrated that STC-1-induced JNK/c-Jun pathway activation may play an important role in the invasiveness of TNBC cells.

STC-1-induced cell invasion is suppressed by SP600125 in Hs578T TNBC cells. To ascertain the alteration of invasion-related genes such as MMPs by STC-1, we treated Hs578T TNBC cells with $50 \mathrm{ng} / \mathrm{ml}$ STC-1. As shown in Fig. 5A, STC-1 stimulated the levels of MMP-9 expression but not MMP-2 expression. The levels of MMP-9 mRNA expression were increased by $3.28 \pm 0.26$-fold from the control level following STC-1 treatment (Fig. 5A). Under the same condition, STC-1 induced MMP-9 protein expression in the Hs578T TNBC cells (Fig. 5A). Next, we pre-treated Hs578T TNBC cells with SP600125 for $1 \mathrm{~h}$ and then the cells were treated with $50 \mathrm{ng} / \mathrm{ml}$ STC-1. As expected, we observed that SP600125 completely suppressed STC-1-induced JNK phosphorylation in the Hs578T TNBC cells (Fig. 5B). STC-1-induced MMP-9 mRNA expression was decreased to $0.67 \pm 0.02$-fold of the control level by SP600125 (Fig. 5B). Furthermore, we investigated whether the JNK/c-Jun signaling pathway is associated with 
A

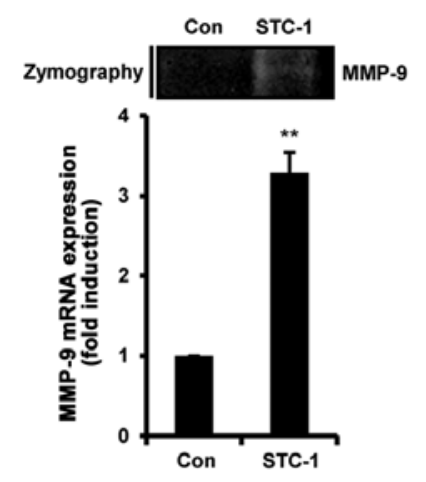

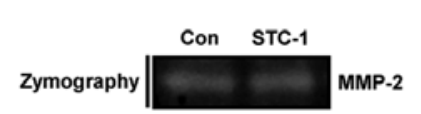

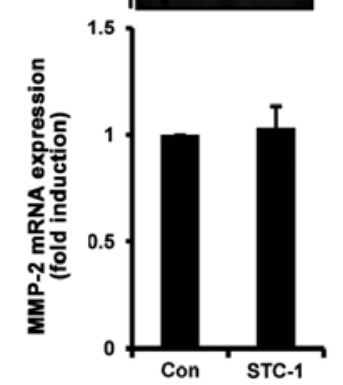

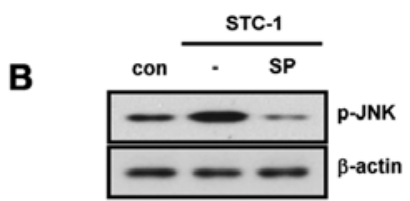

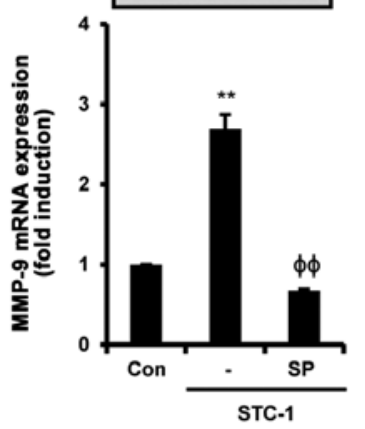

C

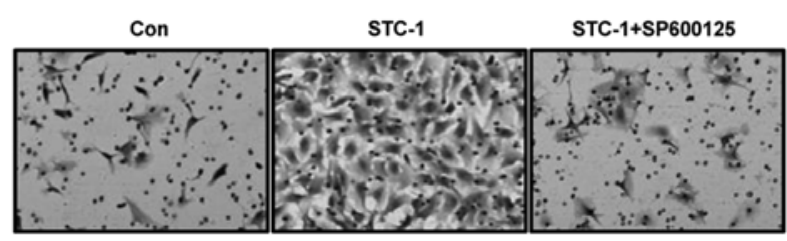

Figure 5. STC-1-induced cell invasion is suppressed by SP600125 in Hs578T TNBC cells. (A) After serum starvation for 24 h, Hs578T TNBC cells were treated with $50 \mathrm{ng} / \mathrm{ml} \mathrm{STC}-1$ for $24 \mathrm{~h}$. The levels of MMP-9 and -2 protein and mRNA expression were analyzed by zymography and real-time PCR, respectively. (B and C) After serum starvation for $24 \mathrm{~h}, \mathrm{Hs} 578 \mathrm{~T}$ TNBC cells were pre-treated with $10 \mu \mathrm{M} \mathrm{SP} 600125$ for $1 \mathrm{~h}$ and then treated with $50 \mathrm{ng} / \mathrm{ml} \mathrm{STC}-1$ for $24 \mathrm{~h}$. (B) The levels of p-JNK and $\beta$-actin expression were analyzed by western blotting. The levels of MMP-9 mRNA expression were analyzed by real-time PCR. (C) The cell invasion rate was analyzed by Boyden chamber assay, as described in 'Materials and methods'. These results are representative of three independent experiments. The values shown are the means \pm SEM. ${ }^{* *} \mathrm{P}<0.01$ vs. the control, ${ }^{\phi} \phi \mathrm{P}<0.01$ vs. the STC-1-treated cells.

STC-1-induced cell invasion in the Hs578T TNBC cells. The induction of cell invasiveness by STC-1 was also significantly decreased by SP600125 (Fig. 5C). Based on these results, we demonstrated that JNK/c-Jun signaling activation by STC-1 triggers cell invasion through upregulation of MMP-9.

\section{Discussion}

STC-1 is highly expressed in a variety of cancers and has prognostic significance in colorectal and gastric cancers $(15,29)$. Abnormal STC-1 expression is associated with poor prognosis in colorectal, esophageal, and gastric cancer patients $(15,29,30)$. However, to date, the clinical significance of STC-1 expression in breast cancer has not been well established. In this study, we investigated the correlation between STC-1 expression and survival of basal-type breast cancer patients as well as the regulatory mechanism of STC-1 in cell invasion. Consistent with previous reports, our results showed that high expression of STC-1 directly influences relapse-free and overall survival of basal-type breast cancer patients. However, the level of STC-1 expression had no relevance in regards to the survival of luminal- and HER2-type breast cancer patients (data not shown). Therefore, we demonstrated that the STC-1 expression level may be a promising prognostic marker in basal-type breast cancer patients.

STC-1 is involved with various biological events such as cell proliferation, migration, invasion, apoptosis, and inflammation in colon, gastric, and cervical cancer $(14,16,31,32)$. In a recent study, we reported that the motility of TNBC cells such as invasion and migration was higher than these abilities in non-TNBC cells through TGF- $\beta 1$ and $-\beta 2$ expression (33).

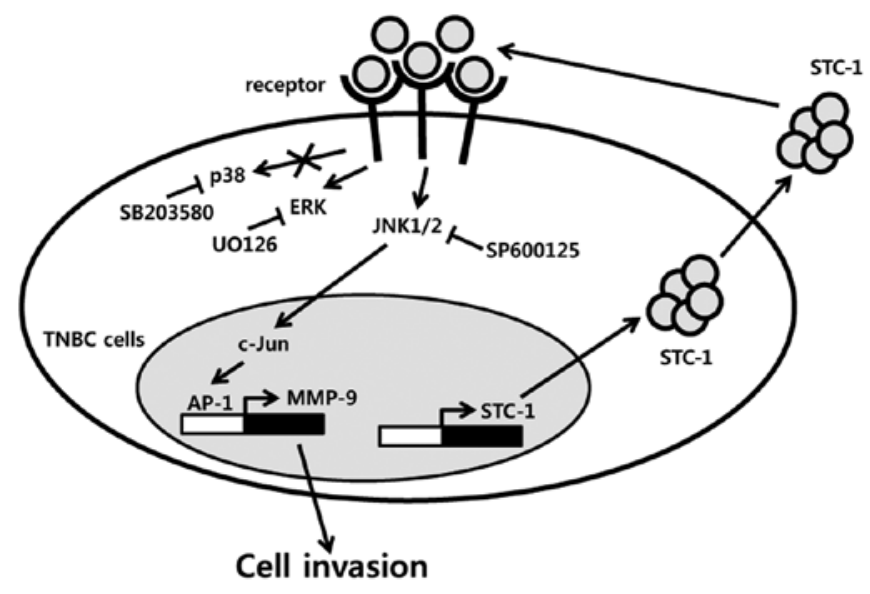

Figure 6. Schematic model. We demonstrated that highly expressed STC-1 protein is secreted to the extracellular matrix and is then incorporated by autocrine/paracrine systems. STC-1/its receptor complex triggers the JNK $\rightarrow$ c-Jun signaling pathway. Activated c-Jun dimers with Fos family and then increases MMP-9 transcriptional activity. Finally, elevated MMP-9 expression enhances the invasiveness of TNBC cells.

Here, we also observed that the levels of STC-1 mRNA and protein expression were significantly increased in the TNBC cells compared with these levels in the non-TNBC cells. Furthermore, our results showed that the invasiveness of TNBC cells was significantly increased by recombinant human STC-1 treatment. In contrast, STC-1 siRNA overexpression suppressed the invasiveness of the TNBC cells. Based on these reports, we demonstrated that STC-1 expression was directly associated with cell invasion in a variety of tumor cells including breast cancer cells. 
Abnormal activities of intracellular signaling molecules such as mitogen-activated protein kinases (MAPKs) in response to extracellular stimuli regulate a variety of cellular activities including cell proliferation, invasion, and transformation (34). Activated MAPKs phosphorylate various substrate proteins including transcription factors such as c-Jun, ATF2, and p53 $(34,35)$. Activation of the JNK/c-Jun signaling pathway is involved with cell invasion and metastasis in ovarian and glioma cancer $(27,28)$. Heterotrimeric G protein, G12, also enhanced cell invasion through the activation of JNK in breast cancer cells (25). Consistent with these reports, we found that STC-1 activated the phosphorylation of JNK and c-Jun in the TNBC cells. In addition, STC-1-induced cell invasion was completely suppressed by a specific JNK inhibitor, SP600125. Therefore, we demonstrated that the STC-1-induced cell invasion was regulated through the JNK/c-Jun-dependent signaling pathway in the TNBC cells.

The aim of this study was to investigate the clinical significance of STC-1 and the regulatory mechanism of STC-1-induced TNBC cell invasion. Aberrant STC-1 expression was associated with poor prognosis in basal-type breast cancer patients. The levels of STC-1 expression and the cell invasiveness were significantly increase in the TNBC cells when compared with that noted in the non-TNBC cells. On the other hand, cell invasiveness of TNBC was decreased by STC-1 siRNA overexpression. Furthermore, STC-1 induced the phosphorylation of JNK and c-Jun in the Hs578T and MDA-MB-231 TNBC cells and then augmented the invasive capacity of the TNBC cells. STC-1-induced cell invasion was suppressed by SP600125 in the TNBC cells. As shown in Fig. 6, we demonstrated that STC-1 enhances the levels of MMP-9 expression through the JNK/c-Jun-dependent signaling pathway and then triggers cell invasion. Thus, we suggest that STC-1 may be a promising therapeutic target for the treatment of patients with TNBC tumors.

\section{Acknowledgements}

This research was supported by the Basic Science Research Program through the National Research Foundation of Korea (NRF) funded by the Ministry of Education (2016R1D1A1B01010508).

\section{References}

1. Siegel R, Naishadham D and Jemal A: Cancer statistics, 2013. CA Cancer J Clin 63: 11-30, 2013.

2. Leclère B, Molinié F, Trétarre B, Stracci F, Daubisse-Marliac L and Colonna M; GRELL Working Group: Trends in incidence of breast cancer among women under 40 in seven European countries: A GRELL cooperative study. Cancer Epidemiol 37: 544-549, 2013.

3. Curtis C, Shah SP, Chin SF, Turashvili G, Rueda OM, Dunning MJ, Speed D, Lynch AG, Samarajiwa S, Yuan Y, et al; METABRIC Group: The genomic and transcriptomic architecture of 2,000 breast tumours reveals novel subgroups. Nature 486: 346-352, 2012.

4. Lehmann BD, Bauer JA, Chen X, Sanders ME, Chakravarthy AB, Shyr Y and Pietenpol JA: Identification of human triple-negative breast cancer subtypes and preclinical models for selection of targeted therapies. J Clin Invest 121: 2750-2767, 2011.

5. Foulkes WD, Smith IE and Reis-Filho JS: Triple-negative breast cancer. N Engl J Med 363: 1938-1948, 2010.

6. Stevens KN, Vachon CM and Couch FJ: Genetic susceptibility to triple-negative breast cancer. Cancer Res 73: 2025-2030, 2013
7. Cleator S, Heller W and Coombes RC: Triple-negative breast cancer: Therapeutic options. Lancet Oncol 8: 235-244, 2007.

8. Chang AC, Jellinek DA and Reddel RR: Mammalian stanniocalcins and cancer. Endocr Relat Cancer 10: 359-373, 2003.

9. Yoshiko Y and Aubin JE: Stanniocalcin 1 as a pleiotropic factor in mammals. Peptides 25: 1663-1669, 2004.

10. Yeung BH, Law AY and Wong CK: Evolution and roles of stanniocalcin. Mol Cell Endocrinol 349: 272-280, 2012.

11. Varghese R, Wong CK, Deol H, Wagner GF and DiMattia GE: Comparative analysis of mammalian stanniocalcin genes. Endocrinology 139: 4714-4725, 1998.

12. Fujiwara Y, Sugita Y, Nakamori S, Miyamoto A, Shiozaki K, Nagano H, Sakon $M$ and Monden $M$ : Assessment of Stanniocalcin-1 mRNA as a molecular marker for micrometastases of various human cancers. Int J Oncol 16: 799-804, 2000.

13. Joensuu K, Heikkilä P and Andersson LC: Tumor dormancy: Elevated expression of stanniocalcins in late relapsing breast cancer. Cancer Lett 265: 76-83, 2008.

14. Liu G, Yang G, Chang B, Mercado-Uribe I, Huang M, Zheng J, Bast RC, Lin SH and Liu J: Stanniocalcin 1 and ovarian tumorigenesis. J Natl Cancer Inst 102: 812-827, 2010.

15. Tamura S, Oshima T, Yoshihara K, Kanazawa A, Yamada T, Inagaki D, Sato T, Yamamoto N, Shiozawa M, Morinaga S, et al: Clinical significance of STC1 gene expression in patients with colorectal cancer. Anticancer Res 31: 325-329, 2011.

16. Block GJ, Ohkouchi S, Fung F, Frenkel J, Gregory C, Pochampally R, DiMattia G, Sullivan DE and Prockop DJ: Multipotent stromal cells are activated to reduce apoptosis in part by upregulation and secretion of stanniocalcin-1. Stem Cells 27: 670-681, 2009.

17. He LF, Wang TT, Gao QY, Zhao GF, Huang YH, Yu LK and Hou YY: Stanniocalcin-1 promotes tumor angiogenesis through up-regulation of VEGF in gastric cancer cells. J Biomed Sci 18: 39, 2011.

18. Du YZ, Gu XH, Li L and Gao F: The diagnostic value of circulating stanniocalcin-1 mRNA in non-small cell lung cancer. J Surg Oncol 104: 836-840, 2011.

19. Lee JM, Dedhar S, Kalluri R and Thompson EW: The epithelial-mesenchymal transition: New insights in signaling, development, and disease. J Cell Biol 172: 973-981, 2006.

20. Liedtke C, Mazouni C, Hess KR, André F, Tordai A, Mejia JA, Symmans WF, Gonzalez-Angulo AM, Hennessy B, Green M, et al: Response to neoadjuvant therapy and long-term survival in patients with triple-negative breast cancer. J Clin Oncol 26: 1275-1281, 2008.

21. Egeblad $\mathrm{M}$ and Werb Z: New functions for the matrix metalloproteinases in cancer progression. Nat Rev Cancer 2: 161-174, 2002.

22. Kim S, Choi JH, Lim HI, Lee SK, Kim WW, Cho S, Kim JS, $\mathrm{Kim} \mathrm{JH}$, Choe JH, Nam SJ, et al: EGF-induced MMP-9 expression is mediated by the JAK3/ERK pathway, but not by the JAK3/STAT-3 pathway in a SKBR3 breast cancer cell line. Cell Signal 21: 892-898, 2009.

23. Han J, Bae SY, Oh SJ, Lee J, Lee JH, Lee HC, Lee SK, Kil WH, Kim SW, Nam SJ, et al: Zerumbone suppresses IL-1 $\beta$-induced cell migration and invasion by inhibiting IL-8 and MMP-3 expression in human triple-negative breast cancer cells. Phytother Res 28: 1654-1660, 2014.

24. Westermarck J, Holmström T, Ahonen M, Eriksson JE and Kähäri VM: Enhancement of fibroblast collagenase-1 (MMP-1) gene expression by tumor promoter okadaic acid is mediated by stress-activated protein kinases Jun N-terminal kinase and p38. Matrix Biol 17: 547-557, 1998.

25. Juneja J, Cushman I and Casey PJ: G12 signaling through c-Jun $\mathrm{NH} 2$-terminal kinase promotes breast cancer cell invasion. PLoS One 6: e26085, 2011.

26. Bachmeier BE,Albini A, Vené R, Benelli R, Noonan D, Weigert C, Weiler C, Lichtinghagen R, Jochum M and Nerlich AG: Cell density-dependent regulation of matrix metalloproteinase and TIMP expression in differently tumorigenic breast cancer cell lines. Exp Cell Res 305: 83-98, 2005.

27. Chang MC, Chen CA, Chen PJ, Chiang YC, Chen YL, Mao TL, Lin HW, Lin Chiang WH and Cheng WF: Mesothelin enhances invasion of ovarian cancer by inducing MMP-7 through MAPK/ERK and JNK pathways. Biochem J 442: 293-302, 2012.

28. Zhou X, Hua L, Zhang W, Zhu M, Shi Q, Li F, Zhang L, Song C and Yu R: FRK controls migration and invasion of human glioma cells by regulating JNK/c-Jun signaling. J Neurooncol 110: 9-19, 2012. 
29. Fang Z, Tian Z, Luo K, Song $\mathrm{H}$ and Yi J: Clinical significance of stanniocalcin expression in tissue and serum of gastric cancer patients. Chin J Cancer Res 26: 602-610, 2014.

30. Shirakawa M, Fujiwara Y, Sugita Y, Moon JH, Takiguchi S, Nakajima K, Miyata H, Yamasaki M, Mori M and Doki Y: Assessment of stanniocalcin-1 as a prognostic marker in human esophageal squamous cell carcinoma. Oncol Rep 27: 940-946, 2012.

31. Peña C, Céspedes MV, Lindh MB, Kiflemariam S, Mezheyeuski A, Edqvist PH, Hägglöf C, Birgisson H, Bojmar L, Jirström K, et al: STC1 expression by cancer-associated fibroblasts drives metastasis of colorectal cancer. Cancer Res 73: 1287-1297, 2013.
32. Guo F, Li Y, Wang J, Li Y, Li Y and Li G: Stanniocalcin1 (STC1) inhibits cell proliferation and invasion of cervical cancer cells. PLoS One 8: e53989, 2013.

33. Kim S, Lee J, Jeon M, Nam SJ and Lee JE: Elevated TGF- $\beta 1$ and $-\beta 2$ expression accelerates the epithelial to mesenchymal transition in triple-negative breast cancer cells. Cytokine 75: 151-158, 2015.

34. Dhillon AS, Hagan S, Rath O and Kolch W: MAP kinase signalling pathways in cancer. Oncogene 26: 3279-3290, 2007.

35. Davis RJ: Signal transduction by the JNK group of MAP kinases. Cell 103: 239-252, 2000. 